University of New Orleans

ScholarWorks@UNO

2002

\title{
Synthesis and magnetic properties of CoPt-poly(methylmethacrylate) nanostructured composite material
}

\author{
Jiye Fang \\ Kevin L. Stokes \\ University of New Orleans \\ Jibao $\mathrm{He}$ \\ Weillie L. Zhou \\ University of New Orleans \\ Charles J. O'Connor \\ University of New Orleans
}

See next page for additional authors

Follow this and additional works at: https://scholarworks.uno.edu/phys_facpubs

Part of the Nanoscience and Nanotechnology Commons, and the Physics Commons

\section{Recommended Citation}

J. Appl. Phys 91, 8816 (2002).

This Article is brought to you for free and open access by the Department of Physics at ScholarWorks@UNO. It has been accepted for inclusion in Physics Faculty Publications by an authorized administrator of ScholarWorks@UNO.

For more information, please contact scholarworks@uno.edu. 
Authors

Jiye Fang, Kevin L. Stokes, Jibao He, Weillie L. Zhou, Charles J. O'Connor, and Daniela Caruntu 


\title{
Synthesis and magnetic properties of CoPt-poly(methylmethacrylate) nanostructured composite material
}

\author{
Jiye Fang, ${ }^{\text {a) }}$ L. D. Tung, Kevin L. Stokes, Jibao He, Daniela Caruntu, Weilie L. Zhou, \\ and Charles J. O'Connor \\ Advanced Materials Research Institute, University of New Orleans, Louisiana 70148
}

\begin{abstract}
We have prepared nanometer-sized CoPt particles dispersed in a poly(methyl methacrylate) (PMMA) matrix, as a novel nanostructured magnetic plastic, through a soft chemical processing route. In this work, CoPt nanoparticles were successfully synthesized from a solution phase reduction system in the presence of capping ligands and stabilizing agents at high temperature. The $\mathrm{CoPt}$ nanoparticles were annealed at $400^{\circ} \mathrm{C}$ for $3 \mathrm{~h}$, and were subsequently re-dispersed in methylmethacrylate (monomer). The polymerization was induced by a UV source and the hardness of final product was adjusted by varying the amount of monomeric cross-link agent. Annealed bare CoPt nanoparticles as a "core" material and CoPt-PMMA composite material were characterized by using energy dispersive spectroscopy, transmission electron microscopy, and x-ray diffraction, indicating that we are able to prepare CoPt nanoparticles with $<10 \mathrm{~nm}$ in diameter (after annealing) by employing this high temperature colloidal processing method. Magnetic investigation of this CoPt-PMMA material indicates an intrinsic coercivity of 300 Oe at $300 \mathrm{~K}$ and 1665 Oe at $5 \mathrm{~K}$.

(C) 2002 American Institute of Physics. [DOI: 10.1063/1.1453329]
\end{abstract}

\section{INTRODUCTION}

Magnetic nanocomposites are an important class of advanced functional materials, and have attracted attention in recent years for magnetic applications such as high-density storage media. The search for new inorganic magnetic materials as "core components" in these composites demands particles with nanometer-scale size so that each particle can be considered as a single magnetic domain. In this article, we present a novel chemical route for fabricating quasimonodisperse CoPt nanoparticles at high temperature as well as CoPt-polymer composites. The magnetic properties of both the CoPt core material and composite material will be discussed.

\section{EXPERIMENT}

\section{A. Chemicals}

In this investigation, super hydride $\left[\mathrm{LiB}\left(\mathrm{C}_{2} \mathrm{H}_{5}\right)_{3} \mathrm{H}, 1.0\right.$ $\mathrm{M}$ in tetrahydrofuran (THF)] was used as a reducing agent. Since THF has a low boiling point, it must be completely replaced by another solvent under inert gas before use. ${ }^{1-3}$ The starting materials also include oleic acid (90\%), oleylamine $(70 \%)$, cobalt (II) chloride $(97.0 \%)$, dioctyl ether (99.0\%), di(ethylene glycol) 2-ethylhexyl ether (98\%, hereafter DEGEE), methylmethacrylate $(99.0 \%$, hereafter MMA), 2-hydroxyethyl methacrylate ( $>99.0 \%$, hereafter HEMA), ethylene glycol dimethacrylate $(98.0 \%$, hereafter EGDA), 2,2-dimethixy-2-phenylacetophenone $(99.0 \%$, hereafter "UV-initiator"), tributylphosphine ( $97.0 \%$, hereafter TBP) and platinum (II) acetylacetonate $\left[\mathrm{Pt}\left(\mathrm{C}_{5} \mathrm{H}_{7} \mathrm{O}_{2}\right)_{2}\right.$, $>99.5 \%$. All of the chemicals were purchased from Aldrich with the exception of the platinum acetylacetonate, which was purchased from Alfa Aesar.

\footnotetext{
a) Author to whom correspondence should be addressed; electronic mail: jfang1@uno.edu
}

\section{B. Synthetic procedure}

Preparation of CoPt-polymer nanostructured composites consists of three steps: fabrication of CoPt seeds, synthesis of CoPt nanocrystals and in situ formation of magnetic nanocomposites. In the first step, $5 \mathrm{ml}$ of dioctyl ether solution consisting of $0.7 \mathrm{mg}$ of $\mathrm{CoCl}_{2}+1.2 \mathrm{mg}$ of $\mathrm{Pt}\left(\mathrm{C}_{5} \mathrm{H}_{7} \mathrm{O}_{2}\right)_{2}$ $+50 \mu \mathrm{l}$ of oleic acid in a $25 \mathrm{ml}$ round bottle flask was heated to $200^{\circ} \mathrm{C}$ under argon stream and $0.25 \mathrm{ml}$ solution of super hydride in dioctyl ether $(0.5 \mathrm{M})$ was injected. The mixture was vigorously stirred and kept at $280^{\circ} \mathrm{C}$ for $20 \mathrm{~min}$. In the second step, $\mathrm{CoCl}_{2}$ (anhydrous, $0.1238 \mathrm{~g}$ ), $\mathrm{Pt}\left(\mathrm{C}_{5} \mathrm{H}_{7} \mathrm{O}_{2}\right)_{2}$ $(0.2140 \mathrm{~g})$, oleic acid $(0.25 \mathrm{ml})$, oleylamine $(0.50 \mathrm{ml})$ and dioctyl ether $(50 \mathrm{ml})$ were mixed in a three-neck round bottom flask under argon and the temperature was steadily increased $\left(\sim 5^{\circ} \mathrm{C} / \mathrm{min}\right)$. At $250^{\circ} \mathrm{C}, 5 \mathrm{ml}$ of super hydride in DEGEE $(1.0 \mathrm{M})$ was injected into the flask containing the CoPt seed particles prepared in the first step (warning: gas bubbles will evolve from the hot solution). The black solution was stirred at $280^{\circ} \mathrm{C}$ for an additional 40 min with a refluxing device. Finally, TBP $(0.45 \mathrm{ml})$ was injected into the flask and the heating source was quickly removed. The system was allowed to cool to room temperature naturally under argon. As-prepared $\mathrm{Co} / \mathrm{Pt}$ nanoparticles were collected by centrifugation, solvent washing and vacuum drying. ${ }^{1,4}$ The magnetic CoPt particles were then annealed at $400{ }^{\circ} \mathrm{C}$ for $3 \mathrm{~h}$ under argon. The annealed CoPt powder was re-dispersed into MMA assisted by ultrasonication for 30 min under argon atmosphere. Within a glovebox, this black homogenous suspension was subsequently transferred into an in situ polymerization system containing the following components: MMA (total), $2.6510 \mathrm{~g}$; HEMA, $0.5754 \mathrm{~g}$; EGDA, $0.9856 \mathrm{~g}$; UVinitiator, $0.2003 \mathrm{~g}$; annealed CoPt, 0.0502 g. Polymerization was initiated by UV light ( $365 \mathrm{~nm}, 100 \mathrm{~W})$ and the sample was continuously irradiated for $60 \mathrm{~min}$. 


\section{Characterization}

Morphology and composition of the annealed $\mathrm{CoPt}$ nanoparticles were characterized using transmission electron microscopy (TEM, JEOL 2010) and energy dispersive x-ray spectroscopy (EDS). Magnetic properties measurement and phase characterization of the nanostructured composites were conducted using a superconducting quantum interference device magnetometer (MPMS-5S Susceptometer from Quantum Design) and $\mathrm{x}$-ray powder diffractometer $(\mathrm{Cu} K \alpha$, Philips X'pert-systems), respectively.

\section{RESULTS AND DISCUSSION}

\section{A. Formation of nanoparticles and structural properties}

In this work, dioctyl ether as a high boiling point, nonpolar organic solvent was chosen as a reaction medium. Cobalt salt and platinum salt with a molar ratio of $1.75: 1$ were dissolved in this organic phase, and reduced by super hydride at high temperature. A similar procedure was used by Sun et al. for preparing Co nanocrystals ${ }^{1}$ and FePt nanocrystals. ${ }^{2}$ In order to allow the nanoparticles to grow large enough, we used a small amount of DEGEE (DEGEE/dioctyl ether $\leqslant 1: 10$ ) as the solvent medium of super hydride, not only because of the excellent solubility of DEGEE in dioctyl ether, but also due to its high boiling point. Nevertheless, a higher concentration of polar DEGEE will collapse this high temperature colloidal system. The as-prepared CoPt colloids were stabilized by a combination of organic ligands (oleic acid and oleyamine). The presence of CoPt seeds may help to form nanoparticles with better quality after the reduction as reported for $\mathrm{Ag}$ nanoparticles. ${ }^{5}$ In order to protect the as-prepared $\mathrm{CoPt}$ nanoparticles against possible agglomeration in later stage and to decrease the tendency of oxidation, we also introduce TBP, an excellent capping agent for Cocontaining colloids. ${ }^{1,2,6}$ However, our results indicate that TBP limits the particle growth if it is introduced too early.

Using a light scattering technique the mean radius of unannealed $\mathrm{CoPt}$ particles was measured as $6.3 \mathrm{~nm}$, and no agglomerate has been detected. Figure 1(a) is a TEM image, showing the morphology of CoPt nanoparticles annealed at $400{ }^{\circ} \mathrm{C}$ for $3 \mathrm{~h}$. It reveals that the particles are virtually uniform in size and are elongated, not spherical. The size of particles covers a range from 4 to $7 \mathrm{~nm}$ in diameter. Nominal elemental analysis was also conducted using an energy dispersive analysis by $\mathrm{x}$-ray (EDS) attachment on the same TEM instrument. Characteristic peaks of $\mathrm{Co}$ and $\mathrm{Pt}$ were present, and the average molar ratio of $\mathrm{Co} / \mathrm{Pt}$ was determined as 46:54. To study phase development of the annealed $\mathrm{CoPt}$ nanoparticles, sample was also analyzed using x-ray powder diffraction (XRD) technique at room temperature. The XRD trace of the annealed CoPt particles, illustrated in Fig. 1(b), indicates that the $\mathrm{CoPt}$ phase is close to quasicubic crystal (tetragonal structure with $a=3.803, c=3.701$ and $C$ $=0.9732$ ) according to the standard ICDD pair distribution function (PDF) (Card No. 43-1358).

Due to the ultrafine size, the annealed CoPt particles could still be dispersed into MMA when assisted by ultrasonication. Polymerization was subsequently conducted by

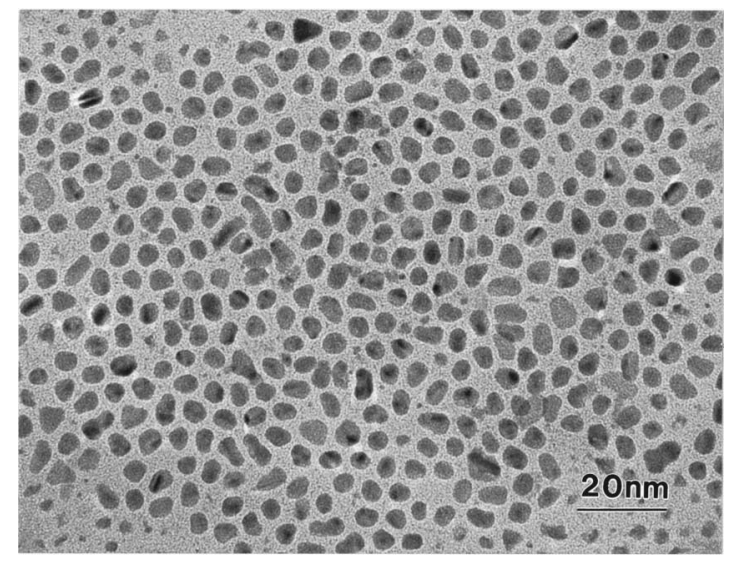

(a)

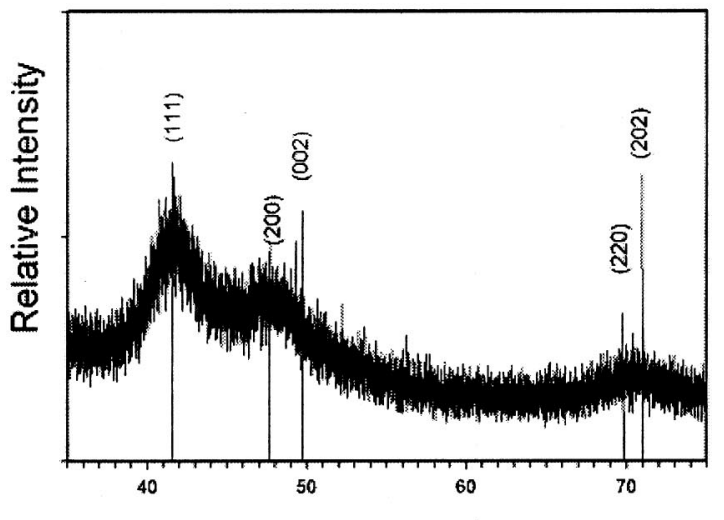

(b)

$2 \theta$ (degree)

FIG. 1. (a): Transmission electron micrograph of bare CoPt nanoparticles annealed at $400{ }^{\circ} \mathrm{C}$ for $3 \mathrm{~h}$. (b): XRD trace of CoPt nanoparticles, annealed at $400{ }^{\circ} \mathrm{C}$ for $3 \mathrm{~h}$.

adding the proper amount of co-monomer and cross-link agent as described above. The hardness of the final product can also be adjusted by varying the amount of monomeric cross-link agent.

\section{B. Magnetic properties}

The zero-field cooled (ZFC) and field cooled (FC) magnetization curves of the composite CoPtpoly(methylmethacrylate) (PMMA) are plotted in Fig. 2. Up to $350 \mathrm{~K}$, we do not observe any blocking temperature $\left(T_{B}\right)$ and the sample remains ferromagnetic to above room temperature. Our results here are different from a previous report in which the blocking temperature was determined as $140 \mathrm{~K}$ for $18 \mathrm{~nm}$ bare CoPt (spherical) particles synthesized in a room-temperature process. ${ }^{7}$ It is worth mentioning that the size of the CoPt particles in our sample is about 4-7 nm and, as mentioned above, the particles are not spherical. It is known that for a magnetic particle system with interaction, the $T_{B}$ (temperature of maximum magnetization ${ }^{8}$ ) can be estimated by ${ }^{9,10}$

$$
T_{B} \approx\left(E_{a}+E_{\mathrm{int}}\right) / 30 k_{B},
$$

where $E_{a}$ is the anisotropy energy and $E_{\text {int }}$ inter-particle interaction energy. The small size of CoPt particles should give rise to the reduction of the energy barrier, which for the uniaxial symmetry is equal to $K V$ (where $K$ is the magnetic 


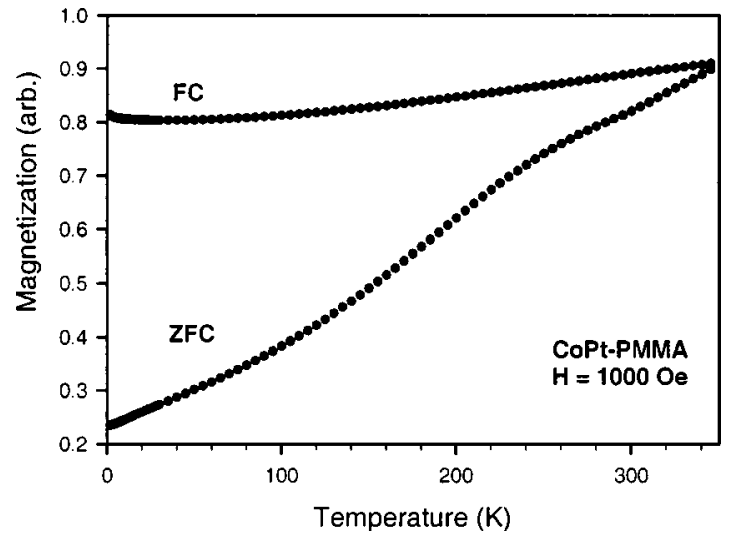

FIG. 2. ZFC and FC of composite CoPt-PMMA measured at the applied field of $1000 \mathrm{Oe}$.

anisotropy constant and $V$ the particle volume), and hence reduces the blocking temperature. Another similar effect is the polymerization, which leads to the significantly weaker inter-particle interaction compared with the bare $\mathrm{CoPt}$ sample and hence decreases the energy barrier $E_{\text {int }}{ }^{11}$ and the blocking temperature as well. Instead of the size and polymerization effects, therefore, the higher blocking temperature in our sample may be attributed to the influence of particle morphology, i.e., the irregular shape, structural orderdisorder difference ${ }^{12}$ and the different synthetic technique as well, resulting in a different particle surface chemistry.

The hysteresis loops at $300 \mathrm{~K}$ of annealed CoPt sample before (a) and after (b) polymerization are plotted in Fig. 3. $\mathrm{Up}$ to the field measured of $5 \mathrm{~T}$, the saturation moment is about $1.5 \mu_{B} / \mathrm{CoPt}$ at., which is significantly lower than that of 1.94 reported for stable CoPt film, ${ }^{13}$ and the reduced remanence of our sample, $M_{r} / M_{s}$, is about 0.2 . The large difference between the saturation magnetization of our nanocrystals, $M_{s}$, and that of the stable thin film, is attributed to the strong interaction between the carbonyl residue of the coating agent and the Co atoms at the particle surface. This effect was calculated and observed for small $\mathrm{Ni}$ and NiPt clusters coated by Co ligands. Carbonyl ligands completely quench the magnetic moments of the $\mathrm{Ni}$ atoms at the cluster surface, leaving the inner core metal atoms unaffected. ${ }^{14}$

Our bare CoPt sample (after annealing) exhibits an intrinsic coercivity $\left(H_{c}\right)$ of $260 \mathrm{Oe}$ at $300 \mathrm{~K}$. The CoPt sample dispersed in PMMA gives $H_{c}$ of 300 Oe at $300 \mathrm{~K}$, and 1665 Oe at $5 \mathrm{~K}$. The lower value of $H_{c}$ for the bare CoPt sample compared with the polymerized composite is interesting. The stronger inter-particle interaction in the unpolymerized sample may act to reduce the coercivity or ${ }^{15}$ it may be due to the difference in the surface chemistry between the polymerized and unpolymerized samples. Moreover, it has been reported that CoPt can be crystallized in two different phases: the (magnetically soft) disordered fcc structure and the (magnetically hard) ordered fct structure. From the x-ray measurement, it is difficult to distinguish between fcc CoPt and fct CoPt phases as their XRD patterns are very similar and all

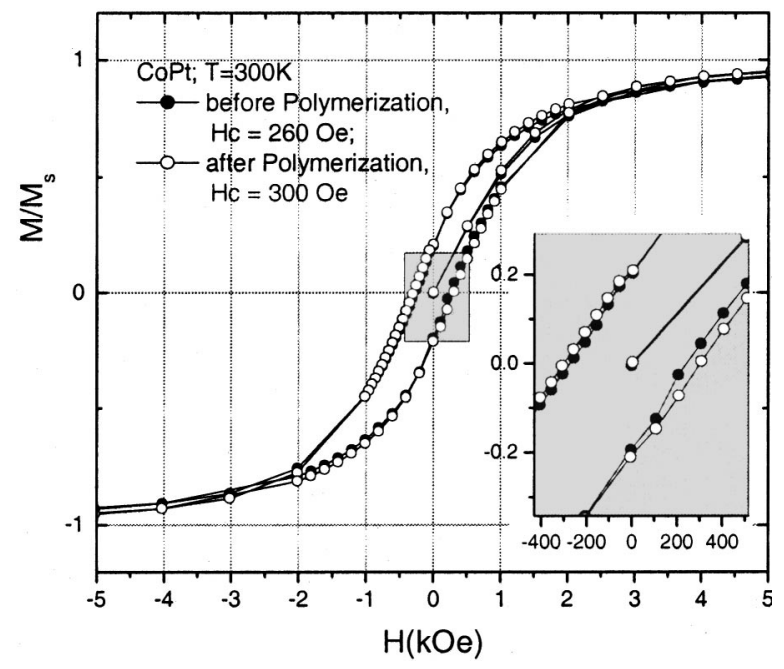

FIG. 3. Hysteresis loop measured at $300 \mathrm{~K}$ of annealed CoPt nanoparticles: (O) before polymerization and $(\bigcirc)$ after polymerization.

the peaks are considerably broadened due to very small particle sizes. ${ }^{16}$ These two different phases, however, can be distinguished from the magnetic measurements. In our CoPt-PMMA sample, the small value of $H_{c}$, i.e., $260 \mathrm{Oe}$ (before polymerization) and 300 Oe (after polymerization), would indicate that the annealed CoPt is crystallized in fcc structure.

\section{ACKNOWLEDGMENTS}

This work was supported by research Grants No. DAAD19-99-1-0001 from the Army Research Office and Grant No. (2001-04)-RII-03 from the LA Board of Regents, NSF/LEQSF.

${ }^{1}$ S. Sun and C. B. Murray, J. Appl. Phys. 85, 4325 (1999).

${ }^{2}$ S. Sun, C. B. Murray, D. Weller, L. Folks, and A. Moser, Science 287, 1989 (2000)

${ }^{3}$ C. B. Murray, C. R. Kagan, and M. G. Bawendi, Annu. Rev. Mater. Sci. 30, 545 (2000).

${ }^{4}$ J. Fang, K. L. Stokes, W. L. Zhou, W. Wang, and J. Lin, Chem. Commun. (Cambridge) 1872 (2001).

${ }^{5}$ N. R. Jana, L. Gearheart, and C. J. Murphy, Chem. Commun. (Cambridge) 617 (2001).

${ }^{6}$ C. B. Murray, S. Sun, W. Gaschler, H. Doyle, T. A. Betley, and C. R. Kagan, IBM J. Res. Dev. 45, 47 (2001).

${ }^{7}$ E. E. Carpenter, C. T. Seip, and C. J. O’Connor, J. Appl. Phys. 85, 5184 (1999).

${ }^{8}$ L. Garcell, M. P. Morales, M. Andres-Vergés, P. Tartaj, and C. J. Serna, J. Colloid Interface Sci. 205, 470 (1998).

${ }^{9}$ J. L. Dormann, L. Spinu, E. Tronc, J. P. Jolivet, F. Lucari, F. D. 'Orazio, and D. Firorani, J. Magn. Magn. Mater. 183, 255 (1998).

${ }^{10}$ D. A. Dimitrov and G. M. Wysin, Phys. Rev. B 54, 2472 (1996).

${ }^{11}$ J. L. Dormann, L. Bessais, and D. Fiorani, J. Phys. C 21, 2015 (1988).

${ }^{12}$ M. P. Morales, M. Andres-Verges, S. Veintmillas-Verdaguer, M. I. Montero, and C. J. Serna, J. Magn. Magn. Mater. 203, 146 (1999).

${ }^{13}$ G. S. Chang, Y. P. Lee, J. Y. Rhee, J. Lee, K. Jeong, and C. N. Whang, Phys. Rev. Lett. 87, 067208 (2001).

${ }^{14}$ D. A. van Leeuwen, J. M. van Ruitenbeck, L. J. de Jongh, A. Ceriotti, G. Pachioni, O. D. Häberlen, and N. Rösch, Phys. Rev. Lett. 73, 1432 (1994).

${ }^{15}$ M. W. Crabbe, H. A. Davies, and R. A. Buckley, IEEE Trans. Magn. 30, 696 (1994).

${ }^{16}$ M. Yu, Y. Liu, A. Moser, D. Weller, and D. J. Sellmyer, Appl. Phys. Lett. 75, $3992(1999)$ 